\title{
ATRIAL FIBRILLATION
}

RF NEEDLE IMPROVES SEPTAL PUNGTURE

The use of a radiofrequency (RF) needle improves the speed, efficacy, and safety of trans-septal catheterization in patients undergoing ablation of the left atrium for atrial fibrillation (AF). These are the findings from a single-center study published in Heart Rhythm. "The introduction of the RF trans-septal needle represents the first major equipment advance for trans-septal puncture in almost 50 years," write the investigators.

"We had done a large number of trans-septal punctures using standard needles," says Dr Roger Winkle, who led the study. "It was always an uncomfortable procedure ... [and] we were on the look-out for better and safer tools." The NRG ${ }^{\circledR}$ RF trans-septal needle was developed by Baylis Medical (Saint-Laurent, QC, Canada) and was used by the investigators for a series of $575 \mathrm{AF}$ ablations. The outcomes of these procedures were compared with the researchers' previous experience of using standard BRK ${ }^{\mathrm{TM}}$ trans-septal needles (St Jude Medical, Maple Grove, MN, USA) for 975 ablation procedures.

Among the 1,167 patients who underwent $\mathrm{AF}$ ablation with either of the two needles, the most prevalent rhythm abnormality was persistent AF (52.9\%). The NRG ${ }^{\circledR}$ needle had a significantly lower failure rate for transseptal puncture than the standard needle (0.17\% vs $1.23 \%, P=0.039)$. The only case in which the RF needle failed to perforate the septum was in a patient with hypertrophic cardiomyopathy. None of the patients undergoing ablation with the RF needle experienced pericardial tamponade, compared with nine of those whose procedure involved the use of the standard needle $(P=0.031)$.

"We hope our work will stimulate others to ... adopt the new RF technology," concludes Dr Winkle. "It should reduce complications from AF ablation and other procedures that require left atrial entry."

Alexandra King

Original article Winkle, R. A., Mead, R. H., Engel, G. \& Patrawala, R. A. The use of a radiofrequency needle improves the safety and efficacy of trans-septal puncture for atrial fibrillation ablation. Heart Rhythm doi:10.1016/ j.hrthm.2011.04.032 